\title{
Discovery of polarized emission from the long period intermediate polar RX J2133.7+5107
}

\author{
S. Katajainen ${ }^{1}$, O. W. Butters ${ }^{2}$, A. J. Norton ${ }^{2}$, H. J. Lehto ${ }^{1,3}$, and V. Piirola ${ }^{1,4}$ \\ 1 Tuorla Observatory, University of Turku, Väisäläntie 20 21500, Piikkiö, Finland \\ e-mail: [sekataja;hlehto;piirola]@utu.fi \\ 2 Department of Physics and Astronomy, The Open University, Walton Hall, Milton Keynes MK7 6AA, UK \\ e-mail: [o.w.butters; A.J.Norton]@open.ac.uk \\ 3 Department of Physics, 20014 University of Turku, Finland \\ 4 Vatican Observatory, 00120 Cittá del Vaticano
}

Received 6 April 2007 / Accepted 18 September 2007

\section{ABSTRACT}

\begin{abstract}
Aims. We intended to investigate the magnetic field properties of the recently identified intermediate polar RX J2133.7+5107.
Methods. We carried out UBVRI photopolarimetric observations of the target using TURPOL on the Nordic Optical Telescope over 2 nights in July/August 2006.

Results. We found that RX J2133.7+5107 emits circularly polarized light in all UBVRI bands (up to 3\%). This is the first detection of circular polarization in this object. The circular polarization modulations and flux variations give hints of cyclotron beaming effects and suggest that the field strength in RX J2133.7+5107 is possibly one of the highest found amongst the IPs.

Conclusions. The highly asynchronous rotation of RX J2133.7+5107 (the spin to orbital period ratio is $\sim 0.022$ ), suggests that it has only recently come into contact and although it is likely to evolve into a polar, it is currently a long way from doing so. We suggest a possible link between the detection of a soft X-ray blackbody component and polarized optical emission in intermediate polars.
\end{abstract}

Key words. stars: binaries: close - stars: magnetic fields - polarization - stars: novae, cataclysmic variables stars: individual: RX J2133.7+5107

\section{Introduction to magnetic cataclysmic variables}

Cataclysmic Variables (CVs) are binary stars where a low mass main sequence star (the secondary) transfers matter via Roche lobe overflow onto a compact white dwarf (WD) primary. One group of $\mathrm{CVs}$, among their many subclasses, is the magnetic CVs (mCVs) (Cropper 1990). In these systems the WD has a strong enough magnetic field to channel the accreting matter along the magnetic field lines toward the magnetic poles near the surface of the WD. Just above the magnetic poles the accretion flow forms a strong shock, as accreted matter is decelerated from supersonic to subsonic speeds. This matter then cools, emitting hard X-rays ( $k T \sim 10-30 \mathrm{keV})$ as bremsstrahlung radiation and cyclotron radiation in the optical and infrared (Cropper 1990; Warner 1995).

In mCVs where the magnetic field strength is of the order tens or even hundreds of MG, ranging from 7 MG (V2301 Oph, Ferrario et al. 1995) to 230 MG (AR UMa, Schmidt et al. 1996), the strong magnetic field prevents the formation of an accretion disc, and the matter follows the field lines from the inner Lagrange point down to the surface of the WD. In addition, the magnetic locking torque balances the accretion torque and the spin of the WD is synchronized with the orbital period (typically $\leq 2 \mathrm{~h}$ ). These systems are known as polars.

Those mCVs with a slightly weaker magnetic field $(B \leq$ $10 \mathrm{MG}$ is generally assumed, see Warner 1995) do generally possess an accretion disc. In these systems, known as intermediate

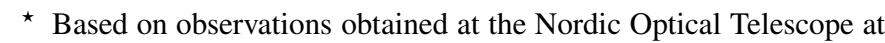
the Roque de los Muchachos Observatory in La Palma. polars (IPs), the WDs typically have spin periods of the order of $\sim 1000 \mathrm{~s}$ and are asynchronous with the orbit, which typically has a period of a few hours. The subclass of rapidly spinning IPs, with spin periods from $31 \mathrm{~s}$ (AE Aqr) to of order a hundred seconds (DQ Her: $142 \mathrm{~s}$ ) are occasionally referred to as DQ Her stars, but often in the literature all IPs are referred to as DQ Her stars (for a comprehensive review of intermediate polars see Patterson 1994).

Many IPs have a WD spin period which is about one tenth of the orbital period of the binary system and this has long been suspected to be the case in IPs in general. If IPs are in spin equilibrium, a period ratio of about 0.1 is expected for typical mass ratios, as long as the co-rotation radius (i.e. that at which material in a local Keplerian orbit co-rotates with the magnetic field of the white dwarf) is at the circularization radius (i.e. that at which the specific angular momentum equals that of matter at the inner Lagrangian point). However, in the last 5 years, several IPs with rather different spin/orbital period ratios have been found, e.g 1RXS J173021.5-055933 $\left(P_{\text {spin }} / P_{\text {orb }}=0.0023\right.$, Gaensicke et al. 2005); HS2331+3905 $\left(P_{\text {spin }} / P_{\text {orb }}=0.0138\right.$, Araujo-Betancor et al. 2005); 1RXS J1548-4528 (NY Lup) $\left(P_{\text {spin }} / P_{\text {orb }}=0.0195\right.$, Haberl et al. 2002; de Martino et al. 2006a); Swift J0732.5$1331 \quad\left(P_{\text {spin }} / P_{\text {orb }}=0.0254, \quad\right.$ Patterson et al. 2006); 1RXS J070407.9+262501 $\left(P_{\text {spin }} / P_{\text {orb }}=0.0320\right.$, Gaensicke et al. 2005); HS0943+1404 $\left(P_{\text {spin }} / P_{\text {orb }}=0.276\right.$, Rodrigues-Gil et al. 2005); DW Cnc $\left(P_{\text {spin }} / P_{\text {orb }}=0.448\right.$, Patterson et al. $2004) ; \quad \operatorname{SDSS} \quad \mathbf{J} 233325.92+152222.1 \quad\left(P_{\text {spin }} / P_{\text {orb }}=0.501\right.$, Southworth et al. 2007) and SDSS J023322.61+005059.5 $\left(P_{\text {spin }} / P_{\text {orb }}=0.625\right.$, Southworth et al. 2006). In the most 
extreme cases, such as AE Aqr, the spin to orbital period ratio is as small as $\sim 0.001$.

On the other hand, some polars are known to have a WD spin period which differs by a few percent from their orbital period: V1500 Cyg (Stockman et al. 1988), BY Cam (Silber et al. 1992), V1432 Aql (RX J19402-1025) (Patterson et al. 1995; Geckeler \& Staubert 1997), CD Ind (RX J2115-58) (Schwope et al. 1997), and RX J0524+42 (Schwarz et al. 2004). The polar $\mathrm{RX} \mathrm{J0524+42}$ is a key target for studies in $\mathrm{mCV}$ evolution as it could be the first transition object between the IPs and polars where the white dwarf is currently in the process of synchronization. In addition to this, the polars WW Hor and DP Leo have shown secular shifts in their accretion regions, which may be due to small asynchronisms in these binaries (Bailey et al. 1993). One of these asynchronous polars has signs of a recent nova outburst (V1500 Cyg), which may explain the unsynchronized nature of the WD spin period in that case. Although a similar cause might be postulated in the other asynchronous polars, none of them have direct evidence of recent nova explosions.

Polars are predominantly soft X-ray emitters, whilst almost all IPs show quite a hard X-ray spectrum. This has been reinforced by the recent discovery that IPs form a significant population amongst the catalogue of INTEGRAL sources (Barlow et al. 2006). Recently, however, Evans \& Hellier (2007) have argued that most IPs show a soft blackbody component too. Whilst the soft component in Polars is believed to be due to blobby accretion from the stream directly heating the WD surface, Evans $\&$ Hellier (2007) suggest that in IPs this component arises from reprocessing of hard X-rays and its visibility depends on geometric factors in a given system.

Only five IPs have been found to emit polarized light, whereas in all polars the emitted light is both linearly and circularly polarized, due to cyclotron emission processes near the surface of the WD. Those IPs where circular polarization has been found so far are: BG CMi (Penning et al. 1986; West et al. 1987), PQ Gem (RE 0751+14) (Rosen et al. 1993; Piirola et al. 1993; Potter et al. 1997), V2400 Oph (RX J1712.6-2414) (Buckley et al. 1997), V405 Aur (RX J0558.0+5353) (Shakhovskoj \& Kolesnikov 1997) and V2306 Cyg (1WGA J1958.2+3232) (Uslenghi et al. 2001; Norton et al. 2002).

As the weakest magnetic fields found in polars have been estimated to be $\sim 7 \mathrm{MG}$ (V2301 Oph, i.e. 1H1752+08, Ferrario et al. 1995), and the strongest magnetic field strengths in IPs have been estimated as more than $10 \mathrm{MG}$ (i.e. PQ Gem: 818 MG (Piirola et al. 1993) or 9-21 MG (Vaeth et al. 1996; Potter et al. 1997) and V2400 Oph: 9-20 MG (Vaeth 1997)), there is likely to be some overlap between the magnetic field strengths in these two subclasses. It is not clear though how the evolution of IPs and polars is connected. Polars are predominantly seen at shorter orbital periods $\left(P_{\text {orb }}<2 \mathrm{~h}\right)$ and intermediate polars at longer orbital periods $\left(P_{\text {orb }}>3 \mathrm{~h}\right)$. However, there are several examples of each type of system outside these ranges, and the period distribution of $\mathrm{mCVs}$ as a whole may not exhibit the $\sim 2-3 \mathrm{~h}$ period gap, as seen in non-magnetic systems Wickramasinghe \& Wu (1994).

All CVs will evolve towards shorter orbital periods as they lose angular momentum via magnetic wind braking and gravitational radiation. In $\mathrm{mCVs}$, the magnetic white dwarfs are also expected to spin down on long timescales, under the effect of the magnetic locking torque. Chanmugam \& Ray (1984) consequently suggested that polars evolve from IPs, but this idea has not generally been accepted, mostly because very few IPs are found to emit circularly polarized radiation. Polarization surveys, such as that by Cropper (1986), have shown that upper limits to the fractional circular polarization in many IPs are around $0.1-0.2$ per cent. It is therefore generally believed that the magnetic field strength in IPs is about 10-100 times lower than that found in polars, so evolving one subclass into the other may not be feasible. Cumming (2004) suggested a possible solution whereby the relatively high accretion rates in IPs may overcome ohmic diffusion, such that magnetic flux is advected into the interior of the white dwarf, reducing the surface magnetic field strength. This effective burying of the WD magnetic field would make them appear less magnetic than they really are. In this case there is also so much unpolarized light (due to the high accretion rate) that polarized emission is not seen. Under his suggestion, once accretion turns off below an orbital period of $\sim 3 \mathrm{~h}$, the magnetic field resurfaces, so that when accretion resumes below an orbital period of $\sim 2 \mathrm{~h}$, the system will synchronize and emerge as a polar.

The magnetic moment of the WD, $\mu_{1}$, seems to determine whether a mCV will appear as an IP, a polar, or just as a nonmagnetic (i.e. "normal") CV. According to Norton et al. (2004), there exists a difference in the observed magnetic moments of polars and IPs, with virtually all IPs having $\mu_{1} \leq 5 \times 10^{33} \mathrm{G} \mathrm{cm}^{3}$ and all polars having $\mu_{1} \geq 5 \times 10^{33} \mathrm{G} \mathrm{cm}^{3}$. It is estimated (King et al. 1985; Lamb \& Melia 1987; Norton et al. 2004) that if $\mu_{1} \leq 10^{31} \mathrm{G} \mathrm{cm}^{3}$, the magnetic field of the WD is not able to funnel the accretion flow, and we do not then observe any evidence for magnetic fields. If $10^{31} \mathrm{G} \mathrm{cm}^{3} \leq \mu_{1} \leq 5 \times 10^{33} \mathrm{G} \mathrm{cm}^{3}$ and $P_{\text {orb }}>3 \mathrm{~h}$, a magnetic CV will be seen as an IP, which may evolve eventually into a low magnetic field polar or if their magnetic field is stronger than supposed (under the model of Cumming (2004) mentioned above), they could evolve into polars below the $\sim 2-3 \mathrm{~h}$ period gap. Finally, if $\mu_{1} \geq 5 \times 10^{33} \mathrm{G} \mathrm{cm}^{3}$ and $\mathrm{P}_{\text {orb }}>3 \mathrm{~h}$, an IP will rapidly evolve into a polar in the conventional manner.

\section{The intermediate polar $\mathrm{RX} \mathbf{J} 2133.7+5107$}

In this paper we discuss our recent UBVRI photopolarimetric observations of the long period intermediate polar RX J2133.7+5107. This target was discovered from the ROSAT Galactic Plane survey by Motch et al. (1998), and identified as a $B \sim 16$ mag star, which they classified as a CV. Recent observations with XMM-Newton showed that the X-ray data from RX J2133.7+5107 require a multi-temperature bremsstrahlung and a blackbody component with a temperature $k T_{\mathrm{bb}} \sim 100 \mathrm{eV}$ in order to fit the detailed spectrum obtained (de Martino et al. 2006b). It is therefore similar to other IPs with a strong soft X-ray spectral component such as PQ Gem (RE 0751+14, Duck et al. 1994), V405 Aur (RX J0558.0+5353, Haberl \& Motch 1995), UU Col (RX J0512.2-3241, Burwitz et al. 1996), NY Lup (RX J154814.5-452845, Haberl et al. 2002) and MU Cam (RX J062518.2+733433, Staude et al. 2003).

Like the soft intermediate polar NY Lup, but unlike most other IPs, RX J2133.7+5107 has an unusually long orbital period, which in this case is $P_{\text {orb }}=7.193 \pm 0.016 \mathrm{~h}$ (BonnetBidaud et al. 2006). Only a few IPs are known to have a longer orbital period than this, and thus RX J2133.7+5107 is among the widest IP binaries. The spin period of the WD in RX J2133.7+5107 is relatively short, $P_{\text {spin }}=570.823 \pm$ $0.013 \mathrm{~s}$, and its $P_{\mathrm{spin}} / P_{\text {orb }}$ ratio (a measure of the degree of asynchronism) is therefore 0.022 , which is one of the smallest amongst all IPs: Norton et al. (2004) Table 1 lists only 7 IPs and IP candidates which have smaller $P_{\text {spin }} / P_{\text {orb }}$ ratio (WZ Sge, AE Aqr, GK Per, V533 Her, DQ Her, XY Ari and V709 Cas), to which may now be added also HS2331+3905 
Table 1. The observing log of RX J2133.7+5107.

\begin{tabular}{rrrrrrrr}
\hline \hline $\begin{array}{r}\text { Date of obs. } \\
\text { (start of night) }\end{array}$ & $\begin{array}{r}\text { HJD of first obs. } \\
(+2453000.0)\end{array}$ & $\begin{array}{r}\text { Durations } \\
(\mathrm{h})\end{array}$ & $\begin{array}{r}\text { Cycles } \\
P_{\text {orb }}\end{array}$ & $P_{\text {spin }}($ full $)$ & Filter(s) & $\begin{array}{r}\text { Exp. Time } \\
(\mathrm{s})\end{array}$ & $\begin{array}{r}(V) \\
(\mathrm{mag})\end{array}$ \\
\hline 2006 July 31 & 948.57384 & 2.01 & 0.28 & 12 & UBVRI & 10 & $15.1-15.5$ \\
2006 August 2 & 950.64636 & 1.81 & 0.25 & 11 & UBVRI & 10 & $14.9-15.4$ \\
\hline
\end{tabular}

(Araujo-Betancor et al. 2005) and 1RXS J154814.5-452845 (NY Lup, de Martino et al. 2006a). On this basis, the magnetic field in RX J2133.7+5107 is expected to be weak (BonnetBidaud et al. 2006). We also note that RX J2133.7+5107 was one of the intermediate polars detected by the INTEGRAL/IBIS survey Barlow et al. (2006) as a hard X-ray (20-100 keV) source.

\section{Observations}

Our observations were made on the nights of 2006 July 31/August 1, and 2006 August 2/August 3 at the $2.56 \mathrm{~m}$ Nordic Optical Telescope (NOT) on La Palma, using TURPOL. This is the Double Image Chopping Polarimeter (Piirola 1973, 1988; Korhonen et al. 1984), which is able to perform simultaneous photopolarimetry in all UBVRI bands by using four dichroic filters (which split the light into five spectral pass-bands). The diaphragm in the instrument has two apertures, one passing the star's light plus the sky background, while the other one passes background light through the aperture. A chopper opens and closes apertures, illuminating the photo-cathode of the photomultiplier tube consecutively. By inserting a plane parallel calcite plate into the beam before the focal plane, polarization measurements are possible. The calcite splits the incoming light into two components, the ordinary and extra-ordinary, which are orthogonally linearly polarized. By measuring the relative intensities of these rays, after a wave-plate, the degree of polarization of the light entering from the star can be measured.

TURPOL was used in its circular-mode, in which intensities are measured in $90.0^{\circ}$ steps of the $\lambda / 4$-plate. For each step, both polarized beams are integrated with a chopping frequency of $25 \mathrm{~Hz}$ for the required integration time. The sky background polarization is directly eliminated using a calcite plate as a polarizing beam splitter. For every orientation of the $\lambda / 4$-plate, a $10 \mathrm{~s}$ integration for each beam is taken. Taking into account some dead time involved in the TURPOL mechanism, integrations take $24 \mathrm{~s}$. One complete polarization observation consists of four different orientation of the $\lambda / 4$-plate (a full $360^{\circ}$ cycle) and the final time resolution is $\sim 1.5 \mathrm{~min}$.

The instrumental polarization and the correct sign of the positive and negative circular polarization were determined through observations of the zero polarization standard star BD +28 4211 and the high circular polarization standard star GRW+70 8247. Instrumental polarization was found to be negligible $(<0.1$ percent) in TURPOL. Sky intensity was measured at $\sim 15 \mathrm{~min}$ intervals. The zero-points of the UBVRI-magnitude scale were determined by using observations of several Landolt standard stars (109 954, 111 250, 1112093 and 114 637; Landolt (1992)) during each night.

\section{Results}

We detected circular polarization from RX J2133.7+5107 in all of the UBVRI wavebands on both observation nights, of order a few percent. Furthermore, there is a modulation at the white dwarf spin period clearly present in both the photometric light curves and in the circular polarization curves. In the following

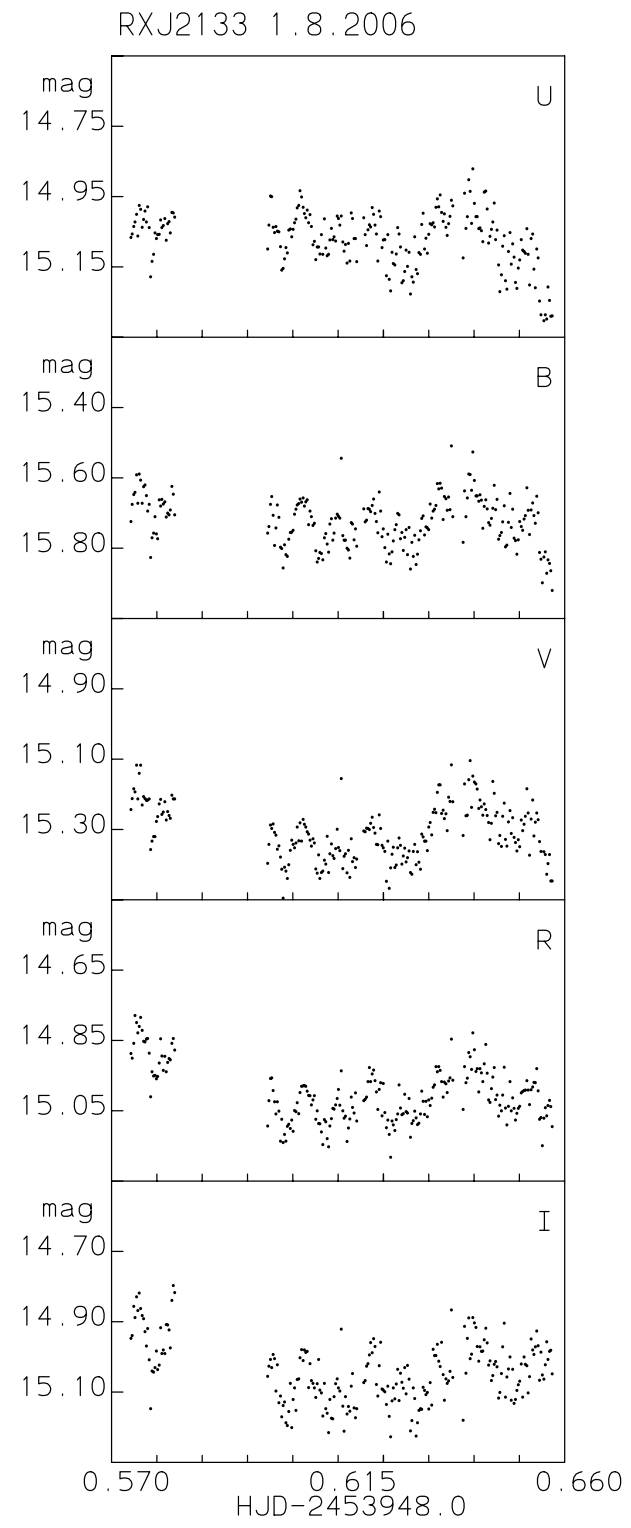

Fig. 1. UBVRI light curves of RX J2133.7 + 5107, obtained on 2006 July 31 /August 1 . Each point presents a single photometric measurement, with $24 \mathrm{~s}$ time resolution.

subsections, we first present the UBVRI light curves and colour indices, followed by the results of the Fourier analysis of the light curves and polarization curves. Finally we present the light curves and polarization curves folded at the white dwarf spin period, using the updated ephemeris which we here define.

\subsection{UBVRI light curves and colour indices}

RX J2133.7+5107 was observed for a total of 3.82 hours over the two nights (see the observing log in Table 1). Figures 1 and 2 show the UBVRI photometry of RX J2133.7+5107 from both 


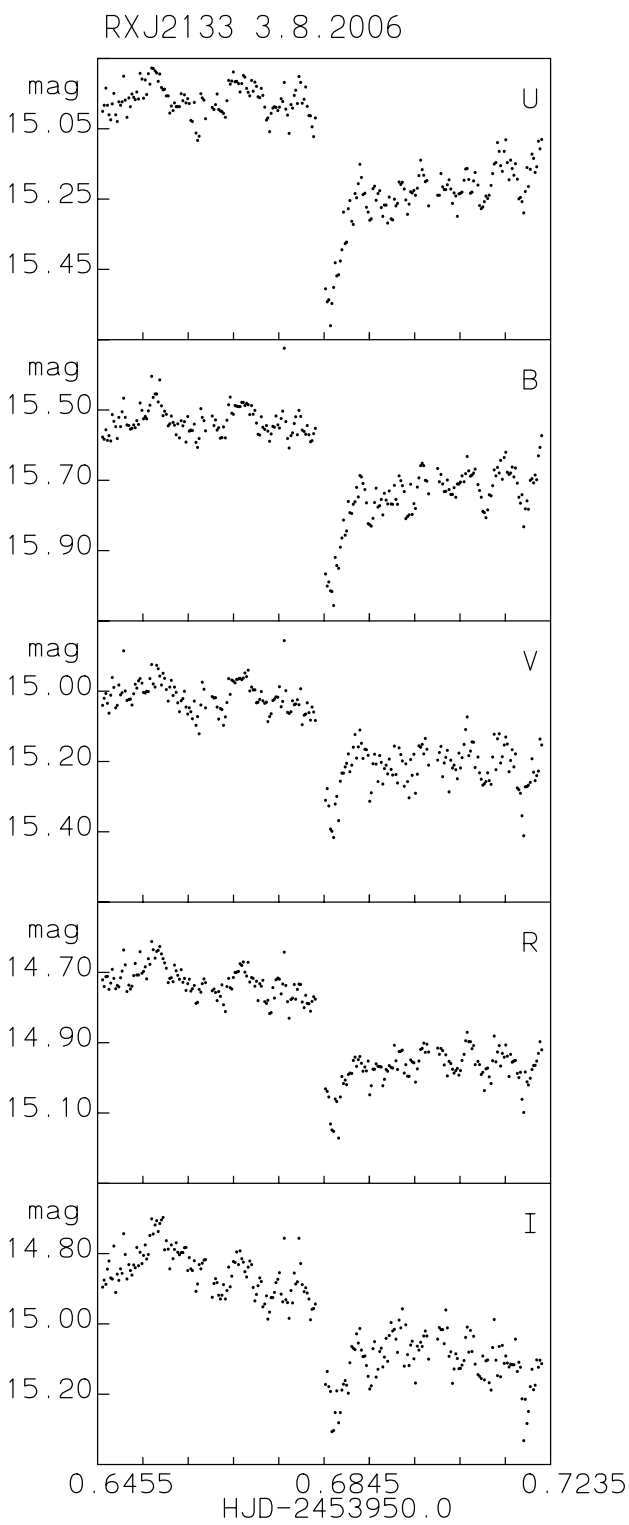

Fig. 2. UBVRI light curves of RX J2133.7 + 5107, obtained on 2006 August 2/August 3 . Each point presents a single photometric measurement, with $24 \mathrm{~s}$ time resolution.

of the observing nights. Each data point corresponds to a single measurement with $24 \mathrm{~s}$ time resolution. This consists of $10 \mathrm{~s}$ integration time on the target, $10 \mathrm{~s}$ integration time on the sky, and $4 \mathrm{~s}$ lost time due to the instrument mechanics. A "saw-tooth" shape with an amplitude of $\sim 0.1$ mag is seen in the light curves, which represents the pulsed modulation at the white dwarf spin period.

On the second night (HJD 2453950.0) a significant dip was observed in all UBVRI bands near the epoch HJD 2453950.685, when the brightness of the target dropped suddenly by approximately 0.5 in the $U$-band, and 0.4 in the $B V R I$-bands. Unfortunately we observed the sky background at the same time as the start of this dip, and therefore missed most of its ingress. Later analysis showed that we were observing RX J2133.7+5107 around same orbital phase as this dip on the first night, but unfortunately no data was taken exactly at the same phase. Bonnet-Bidaud et al. (2006) presented light curves from three separate nights (their Fig. 1) covering almost the whole orbital period $(7.2 \mathrm{~h})$ in each. There is no such dip seen in

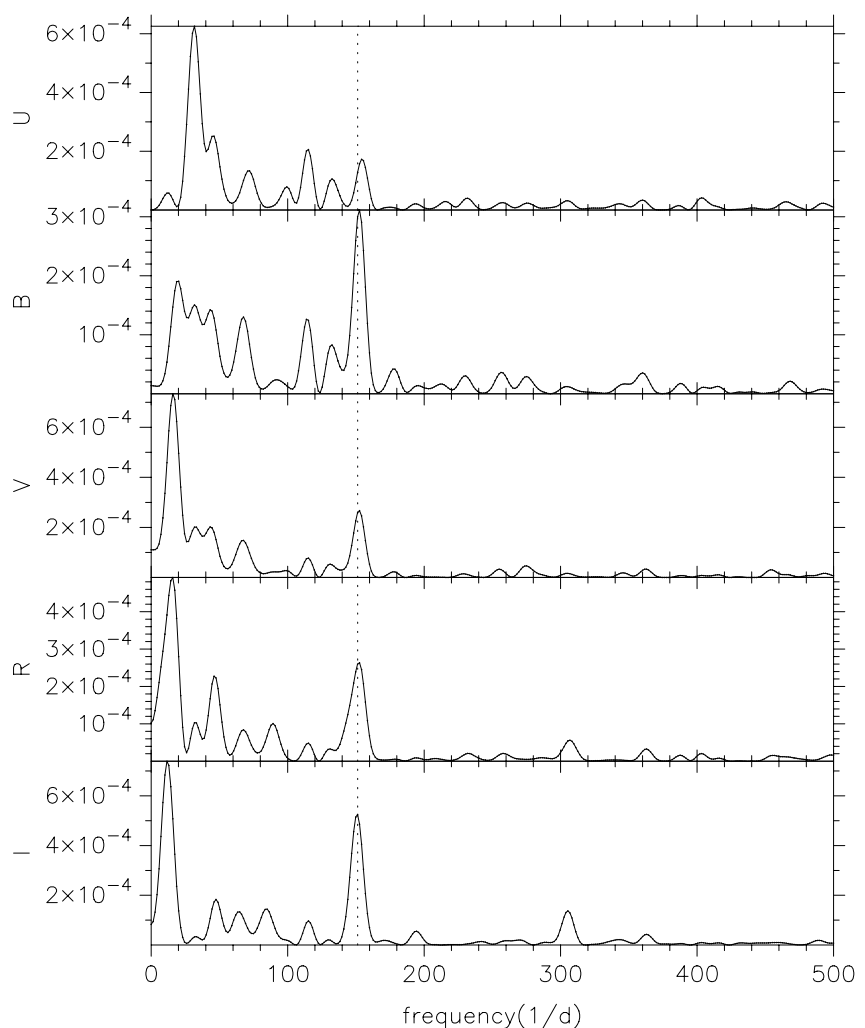

Fig. 3. CLEANed periodogram of the light curves of RX J2133.7+5107 observed during the night of 2006 July 31 /August 1 . The spin frequency of the white dwarf is $\sim 152$ cycles per day. The units of pseudopower on the ordinate axis are $\operatorname{mag}^{2}$. A sinusoid with an amplitude of $A=$ 0.04 mag would produce a peak with power $\sim 4 \times 10^{-4}$.

any of their light curves, and thus the dip seen in our data from 2006 August 2/3, is unlikely to be an eclipse, and its cause still remains a puzzle.

The colours of RX $\mathrm{J} 2133.7+5107$ for $U-B, B-V$ and $V-R$, show some small flickering, and these too exhibit the "saw tooth" profile reflecting the white dwarf pulse period. This is most apparent in the $U-B$ colour.

The average magnitudes in each of the UBVRI bands are $15.1,15.7,15.2,14.9$ and 15.0 respectively. This is consistent with the $B$ band value of 15.8 reported by Motch et al. (1998), indicating that the brightness of the system has not changed significantly.

\subsection{Period analysis of the polarization and light curves}

We performed a CLEAN periodogram analysis for the UBVRI light curves. We selected CLEAN in favour of a more direct, simple power spectrum because in addition to measuring the temporal frequency of the variability, this enabled us to get a good measure of the phases of each component of this variability. On July 31/Aug 1 the single consistent large peak occurred at a frequency of $\sim 152 \mathrm{~d}^{-1}$, which corresponds to the spin period of the white dwarf (Fig. 3).

The details of the analysis are shown in Table 2 . The average frequency of the spin modulation is $152.48 \pm 0.66$ periods per day. This equates to a period of $566.6 \pm 2.5 \mathrm{~s}$, which is within $2 \sigma$ of the value in Bonnet-Bidaud et al. (2006). From our CLEAN analysis, phase zero is defined at the maximum magnitude value of the modulation, and the average phase at the reference time of HJD 2453948.621873 is $-150.75 \pm 7.64$ degrees. This provides 
Table 2. White dwarf spin peaks in the power spectra of RX J2133.7+5107.

\begin{tabular}{rrrr}
\hline \hline & Band & $\begin{array}{r}\text { Frequency } \\
\text { day }^{-1}\end{array}$ & $\begin{array}{r}\text { Zero point of time } \\
\text { HJD -2 453000 }\end{array}$ \\
\hline Night 1 & $U$ & 154.5 & 948.62419 \\
& $B$ & 152.5 & 948.62453 \\
& $V$ & 152.4 & 948.62458 \\
& $R$ & 152.2 & 948.62481 \\
& $I$ & 150.8 & 948.62499 \\
\hline average & 152.48 & 948.62462 \\
Night 2 & 0.66 & 0.00015 \\
& & & \\
& & 163.0 & 950.70601 \\
& $B$ & 155.7 & 950.70596 \\
& $V$ & 156.3 & 950.70594 \\
& $I$ & 158.3 & 950.70614 \\
& 154.5 & 950.70655 \\
\hline averainty & 157.56 & 950.70612 \\
& uncertainty & 1.67 & 0.00013 \\
\hline
\end{tabular}

us with a new zero point for the epoch of maximum magnitude, which takes place at HJD $2453948.62462 \pm 0.00015$. Adopting the more accurate Bonnet-Bidaud et al. (2006) value for the period, we revise the ephemeris by updating only the zeropoint to:

Minimum flux $=$ HJD $2453948.62462(15)+0.0066067(2) \cdot E$.

A second spectral peak in the periodogram is also present at approximately $45 \mathrm{~d}^{-1}$. This corresponds to a period of roughly 0.0222 days or about $32 \mathrm{~min}$. This is roughly the length of the brightening episode seen between $0.628 \mathrm{~d}$ and $0.660 \mathrm{~d}$ in Fig. 1 .

On the night Aug 2/Aug 3, a modulation is also found in all wavebands. The average frequency of $157.56 \pm 1.67$ periods per day can be identified as the spin period of the white dwarf. However, this value is not very trustworthy because it is affected by the very deep dip in the light curve. This dip is also the cause of a consistent feature seen in the power spectra at 253 cycles per day, or a timescale of about 0.004 days.

The time difference between the zeropoints of the two nights equals 315.06 periods, which is consistent with 315 periods, but our data alone cannot exclude other nearby integer numbers of periods. This is the main reason for adopting the Bonnet-Bidaud et al. (2006) period for our ephemeris. Our light curves do not cover the full orbital cycle, so no inference about the orbital period can be made from these data.

An identical analysis was performed for the raw polarization curves. The white dwarf spin frequency is detected clearly in the polarization curves of $R V I$ and only marginally in $U B$ (Fig. 4).

\subsection{UBVRI spin folded circular polarization and light curves}

Figure 5 shows the circular polarimetry obtained at the NOT on the two nights observing, folded at the previously known WD spin period of $570.823 \mathrm{~s}$ and then phase binned into 20 equal bins. Stokes parameters have been calculated for polarization from the integrations made in different orientations of the quarter-wave retarder (rotated in $90.0^{\circ}$ steps). Uncertainties are then calculated both from the photon statistics and from the least square fit to the integrations; whichever is greater has been taken as an uncertainty estimate. The error bars correspond to $\pm 1 \sigma$ uncertainties.

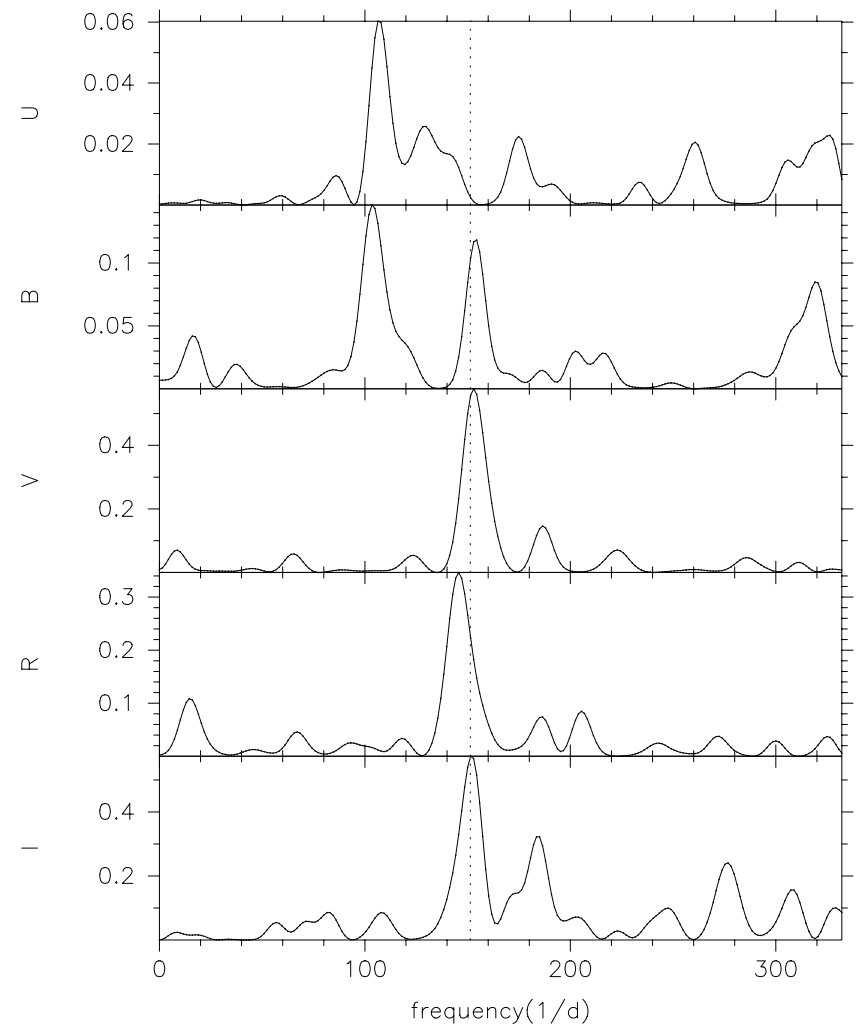

Fig. 4. CLEANed periodogram of the polarization curves of RX J2133.7+5107 observed during the night of 2006 July 31/August 1. The spin frequency of the white dwarf is $\sim 152$ cycles per day. The units of pseudopower on the ordinate axis are $\%^{2}$. A sinusoid with an amplitude of $A=1 \%$ would produce a peak with power $\sim 0.25$.

Significant circular polarization is seen in all UBVRI bands from RX J2133.7+5107, and polarization variations are modulated at the WD spin period. Polarization is near to zero at phase $\Phi=0.5$, and it increases smoothly until relative phase $\Phi=0.0$. After that epoch there is a small dip in the polarization curves in the blue part of the spectrum, near phase $\Phi=0.1-0.2$ (possibly due to cyclotron beaming effects), whilst from phase $\Phi=0.2$ the polarization decreases until $\Phi \sim 0.5$. Polarization is positive over the whole WD spin period, and it has a colour dependence; the peak polarization values in the $U B V R I$ bands are: $+1.5,+2.5$, $+3.5,+3$, and +2.5 percent, respectively.

Figure 6 shows the photometry from the two observing nights, similarly folded and phase binned. Bonnet-Bidaud et al. (2006) observed RX J2133.7+5107 in a broad band (between $300-600 \mathrm{~nm}$ ) filter with a peak at $\sim 380 \mathrm{~nm}$. Reconstructing a broad band filter by combining our corresponding filters allows a direct comparison to be made between our Fig. 7 and their Fig. 4.

\section{Discussion}

\subsection{Magnetic field strength and cyclotron emission}

Known polarized IPs so far have shown polarization predominantly in the red part of their spectrum, for example in PQ Gem, circular polarization is seen only in the $R$ - and $I$-bands in the $U B V R I$ observations of Piirola et al. (1993). Similarly in BG CMi, polarization was found in the red part of the optical and particularly in the infrared region (Penning et al. 1986; West et al. 1987), whilst it was seen in the red part of the spectrum in V405 Aur (Shakhovskoj \& Kolesnikov 1997). 


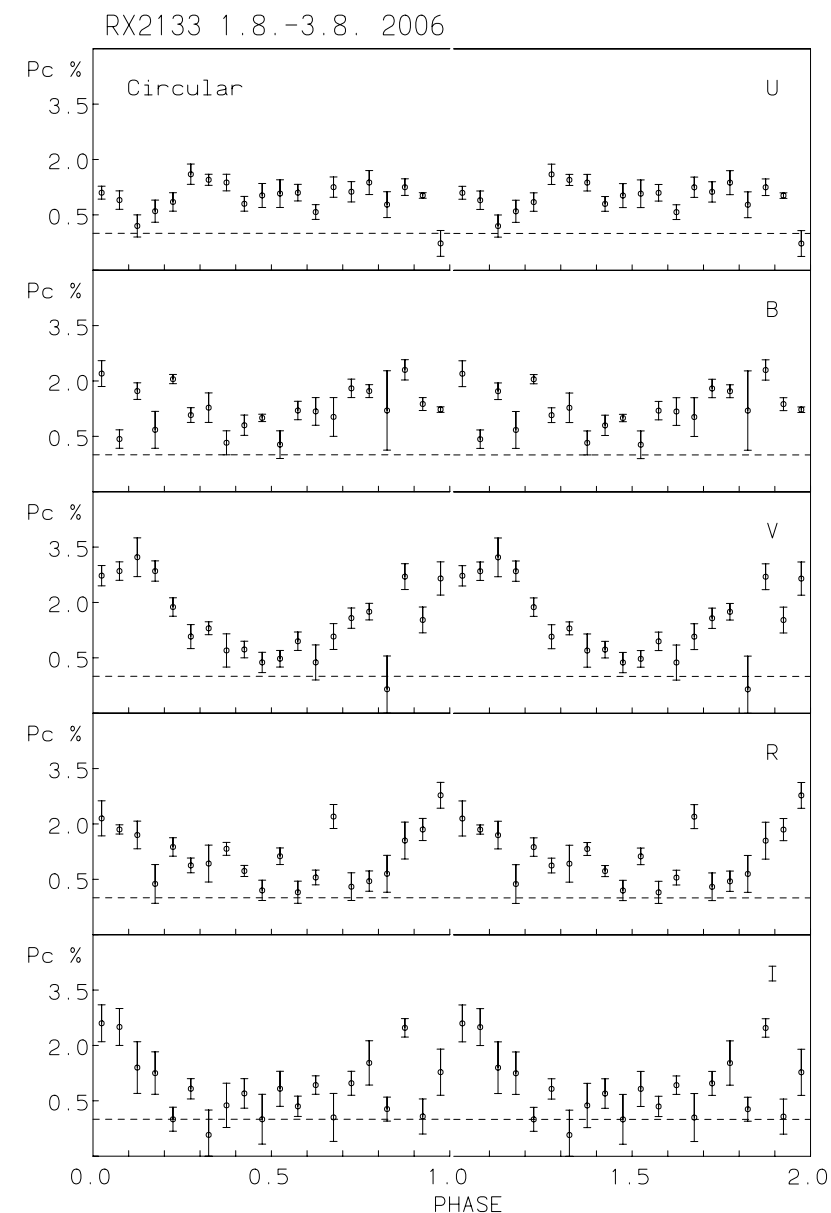

Fig. 5. UBVRI polarization curves of RX J2133.7+5107 observed at the NOT between 2006 July 31 and August 3, folded at the white dwarf spin period of $0.0066067 \mathrm{~d}$ and averaged into 20 phase bins.

Norton et al. (2002) reported optical polarization found in V2306 Cyg, where circular polarization was seen to be negative at the several percent level in the $R$-band, but positive around one percent in the $B$-band. In V2400 Oph, Buckley et al. (1995) found optical polarization which was highest in the $I$ band. RX J2133.7+5107 seems to be quite different in that sense, and this may be an indicator that the inferred magnetic field is stronger than found in other polarized IPs.

In RX J2133.7+5107 circular polarization is positive throughout the spin cycle (Fig. 5), which indicates that one pole is dominant. There is no sign of cyclotron emission from the other (negative) pole. Circular polarization is close to zero near phase 0.5 , which suggests that our line of sight is nearly perpendicular to the magnetic field lines at that phase, i.e. the emission region is at the stellar limb. In terms of the colatitude of the emission region, $\beta$, and the inclination of the WD spin axis, $i$, this can be written as $\beta \sim 90^{\circ}-i$. Circular polarization increases smoothly from phase 0.5 towards 0.0 , when the emission region moves away from the limb to the position where the field lines point closest to us (phase 0.0 ).

At small viewing angles cyclotron emission intensity approaches zero (due to the cyclotron beaming effect), and the diluting effect of unpolarized thermal emission decreases the observed degree of polarization. The peak observed in the $R$ band at phase 0.0 (Fig. 5) becomes flatter in the $V$ band, and a polarization dip is seen in the $B$ and $U$ bands, due to the cyclotron beaming effect, which is strongest at high harmonics (shorter

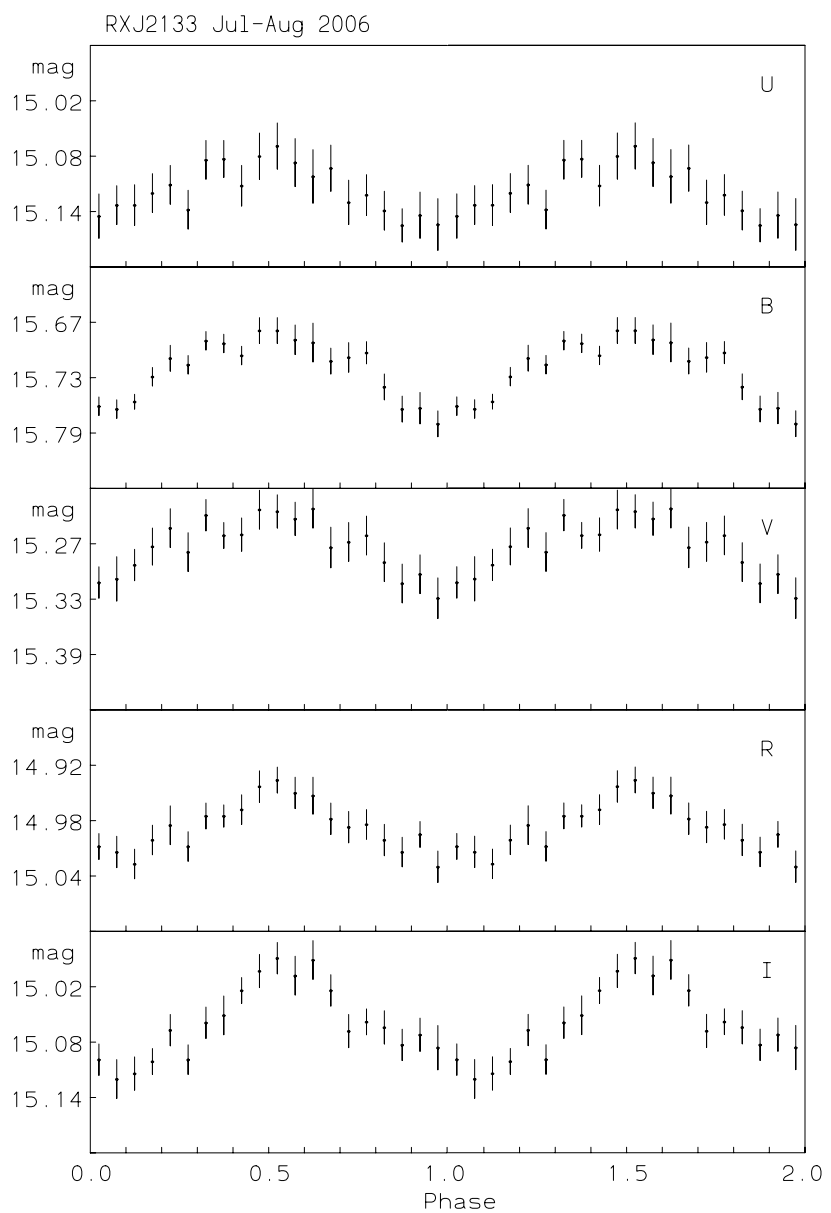

Fig. 6. UBVRI light curves of RX J2133.7+5107 observed at the NOT between 2006 July 31 and August 3, folded at the white dwarf spin period $0.0066067 \mathrm{~d}$ and averaged into 20 phase bins.

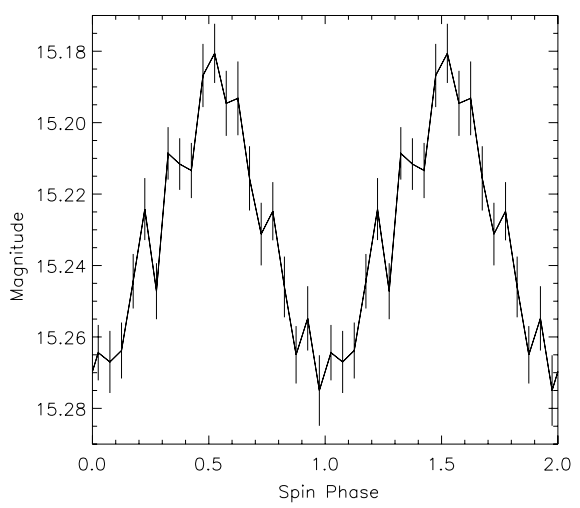

Fig. 7. Reconstructed broad band photometry of RX J2133.7+5107 folded at the $571 \mathrm{~s}$ spin period.

wavelengths). The minimum in the light curves (Fig. 6) takes place near phase 0.0 , in accordance with the proposed cyclotron beaming geometry.

Circular polarization does not go to zero at phase 0.0 , which means that viewing angles are $\gg 0^{\circ}$ even when looking closest along the field lines. Simple geometric considerations then imply that either $i-\beta \gg 0^{\circ}$ or $\beta-i \gg 0^{\circ}$. For a high inclination system the emission region has to be closer to the rotation pole, and for a low inclination system it must be closer to the equator, to fulfil also the relation $\beta \sim 90^{\circ}-i$ (see above). However, without 


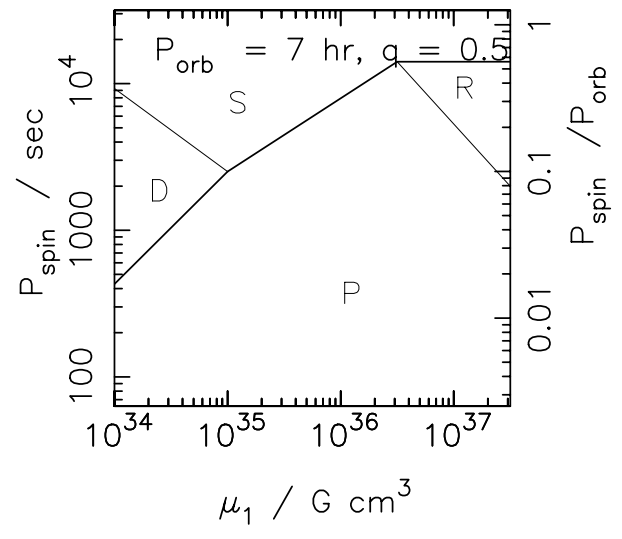

Fig. 8. Spin period vs. magnetic moment diagram for a mass ratio of 0.5 and an orbital period of $7 \mathrm{~h}$ (adapted from Norton et al. (2007). The letters "S", "P", " $D$ " and "R" indicates regions of the parameter space in which a stream-like, propeller-like, disc-like and ring-like flow respectively may be seen. In spin equilibrium, systems tend toward the line which divides disc-like and stream-like flows from propeller-like and ring-like flows.

linear polarization measurements we cannot fix the value of $i$. The absence of eclipses by the companion star requires that $i$ is smaller than about $75^{\circ}$.

Circular polarization variations of RX J2133.7+5107 can be compared with systems where the magnetic field strength is estimated to be about 25MG, such as the polars V834 Cen (Piirola 1995) and BY Cam (Piirola et al. 1994). The observed circular polarization variations in those stars resemble (in their colour dependence) those seen in RX J2133.7+5107. Naturally the circular polarization level is higher in polars, for example in BY Cam or in V834 Cen the circular polarization peak values are almost 30 percent, as there is much less unpolarized light diluting the observed polarization in those systems. In IPs there is more unpolarized light due to their higher accretion rates and larger accretion stream compared to polars. More accurate estimates of the magnetic field of RX J2133.7+5107 could be obtained in the future by using phase resolved circular spectropolarimetry and modelling of such data. Also, high signal-to-noise linear polarimetry of RX J2133.7+5107 to find out the orbital inclination would be very useful.

\subsection{The magnetic moment of $R X J 2133.7+5107$}

Theoretical modelling of IPs by Norton et al. (2004) has begun to explain equilibrium values of the magnetic moment in these systems. They determined the equilibrium spin periods at which systems would lie, with a given orbital period and magnetic moment, assuming the white dwarf not to be spinning up or down on long timescales. This theoretical work has since been extended so that the position of a system in the $P_{\text {spin }} / P_{\text {orb }}$ vs. magnetic moment plane can indicate the structure of the accretion flow in the system (Norton et al. 2007). The key result from this study is that the spin-to-orbital period ratio vs. magnetic moment plane can be divided into regions where the system will display either a disc-like, stream-like, propeller-like or ring-like accretion flow (Fig. 8). Furthermore, the "triple point" positions (at which stream, disc and propeller or stream, ring and propeller flows can co-exist) on the $P_{\text {spin }} / P_{\text {orb }}$ axis are a function of mass ratio and not orbital period. The magnetic moments at which these transitions occur are, however, a function of orbital period.

The mass ratio of RX J2133.7+5107 is uncertain, but likely to be in the range $q \sim 0.3-0.6$ (Bonnet-Bidaud et al. 2006). For a typical mass ratio of 0.5 , the results of Norton et al. (2007) show that, if RX J2133.7+5107 is in equilibrium with $P_{\text {spin }}=571 \mathrm{~s}$ and $P_{\text {orb }} \sim 7 \mathrm{~h}$, its magnetic moment is of order $\sim 10^{34} \mathrm{G} \mathrm{cm}^{3}$; a larger value of magnetic moment for a system with these periods and mass ratio would indicate a propeller-type flow subject to a spin-down of the white dwarf, and a smaller value of the magnetic moment would indicate a disc-like flow which is spinning up the white dwarf (see Fig. 8). For a smaller mass ratio, the magnetic moment corresponding to the equilibrium may be significantly smaller, and for a larger mass ratio, the magnetic moment may be somewhat higher. Hence, for a mass ratio in the range $q \sim 0.3-0.6$, if $\mathrm{RX} \mathrm{J} 2133.7+5107$ is accreting at close to its equilibrium spin rate, the magnetic moment is likely to be in the range $3 \times 10^{33}-3 \times 10^{34} \mathrm{G} \mathrm{cm}^{3}$. This magnetic moment is consistent with a relatively high magnetic field strength, as implied by the polarization results presented earlier.

\subsection{The evolutionary status of $R X J 2133.7+5107$}

RX J2133.7+5107 is apparently a high magnetic field strength intermediate polar (possibly $\mu_{1}$ is about $10^{34} \mathrm{G} \mathrm{cm}^{3}$ ), yet it is a long way from synchronism $\left(P_{\text {spin }} / P_{\text {orb }} \sim 0.022\right)$. A magnetic CV with a field this strong would be expected to synchronize on a relatively short timescale and emerge as a polar with a period of less than about $4 \mathrm{~h}$. However, to become synchronized at the present orbital period it would require an even stronger magnetic field, and in fact very few polars have an orbital period this long: V1309 Ori has the longest known period amongst the polars at $P=7.98 \mathrm{~h}$ (Staude et al. 2001). So we suggest that RX J2133.7+5107 is a relatively young system that has only recently come into contact and begun mass transfer, and has not yet had time to evolve to a shorter orbital period and approach synchronism. Nonetheless we believe it will eventually synchronize and emerge as a polar.

It is also interesting to note a possible link between the presence of significant soft X-ray emission in intermediate polars and the presence of polarized emission, as these two features are also seen in polars. Indeed, Beuermann \& Schwope (1994) demonstrated an anti-correlation between magnetic field strength and the ratio of the strength of the hard X-ray emission to that of the soft X-ray emission in polars. Hence, the polars with stronger magnetic fields have a stronger blackbody component and/or a weaker bremsstrahlung component. In both polars and intermediate polars, a soft X-ray component may arise as accreting material impacts the white dwarf directly, resulting in blackbody radiation with a temperature of $k T_{\mathrm{bb}} \sim 50-100 \mathrm{eV}$ being emitted from the heated white dwarf surface. Although we note the recent suggestion of Evans \& Hellier (2007) that the visibility of a soft component in IPs is a geometrical effect and arises from reprocessing of hard X-rays. In either case, it has a different origin to the multi-temperature X-ray bremsstrahlung emission, whereas the cyclotron emission arises in the cooling plasma as it settles slowly onto the white dwarf surface below a shock. Amongst the soft X-ray emitting IPs, there are two objects which have also been seen to display polarized emission (PQ Gem and V405 Aur) to which may now be added RX J2133.7+5107 as a third example.

We recall the suggestion of Cumming (2004) that the high accretion rates in intermediate polars might overcome ohmic diffusion and significantly affect the magnetic field structure. In this regime, magnetic flux is advected into the interior of the white dwarf, dramatically reducing the surface field. Hence, intermediate polars would appear to have a weaker field than they actually 
have. Now, it is apparent that in some intermediate polars, the accretion flow and magnetic field geometry are such that some of the accreting material directly, or indirectly, heats the white dwarf surface, giving rise to a soft X-ray component. If direct heating occurs, less of the accreting material interacts with the shock, and we suggest this may lead to a less significant "burying" of the white dwarf magnetic field. Hence we might expect those intermediate polars with soft X-ray spectral components to preferentially exhibit signs of stronger magnetic fields, such as enhanced polarized emission.

The intermediate polars with polarized emission that have yet to show evidence for a soft X-ray component (e.g. BG CMi, V2306 Cyg, V2400 Oph) may simply suffer from less significant magnetic field burying for various reasons. Both $\mathrm{BG} \mathrm{CMi}$ and V2400 Oph show evidence for stream-fed accretion (Norton et al. 1992; Buckley et al. 1997) and V2306 Cyg shows evidence for an asymmetric magnetic field at the two poles (Norton et al. 2002). Both of these differences could conceivably reduce the magnetic field burial, allowing detection of polarized emission from accretion under the influence of the inherent (stronger) magnetic field. We suggest that the soft X-ray emitting intermediate polars that are so far without detected polarized emission (e.g. UU Col, MU Cam, NY Lup) would make ideal targets to search for such a component. In particular, the system parameters of NY Lup $\left(P_{\text {spin }}=693 \mathrm{~s}, P_{\text {orb }}=9.87 \mathrm{~h}\right)($ Haberl et al. 2002; de Martino et al. 2006a) make it appear a close twin of RX J2133.7+5107, and it too may be a young intermediate polar which will eventually evolve into a polar.

\section{Conclusion}

We have found that RX J2133.7+5107 emits circularly polarized light in all UBVRI bands (up to $3 \%$ ). The variation of this light gives hints of cyclotron beaming effects. To explain this level of polarization and its colour dependence, a strong magnetic field is needed, (possibly $\mu_{1} \sim 10^{34} \mathrm{G} \mathrm{cm}^{3}$ ), which would make RX J2133.7+5107 one of the most magnetic IPs so far discovered. Due to its highly asynchronous degree of rotation (spin-to-orbital period ratio of 0.022) these results suggest that it may be a young system, which has only recently come into contact. This asynchronism coupled with the inferred magnetic field strength suggests that the system may eventually evolve into a polar.

We propose that there may be a link between the emission of a soft X-ray spectral component due to direct heating of the white dwarf surface and the detection of polarized emission in intermediate polars. Possibly the direct heating means that magnetic field burial below the accretion shock is less effective, so allowing the intrinsic field to produce a stronger polarization signal. If this link is confirmed it would argue that the soft X-ray component in IPs is indeed due to direct heating (as in polars) and not due to reprocessing of the hard X-rays.

Acknowledgements. The Nordic Optical Telescope is operated on the island of La Palma jointly by Denmark, Finland, Iceland, Norway, and Sweden, in the Spanish Observatorio del Roque de los Muchachos of the Instituto de Astrofisíca de Canarias. This work has been supported by the Academy of Finland and "Societas Scientiarum Fennica - Suomen Tiedeseura" and its Magnus Ehrnrooth foundation.

\section{References}

Araujo-Betancor, S., Gaensicke, B. T., Hagen, H.-J., et al. 2005, A\&A, 430, 629 Bailey, J., Wickramasinghe, D. T., Ferrario, L., Hough, J., \& Cropper, M. 1993, MNRAS, 261, L31
Barlow, E. J., Knigge, C., Bird, A. J., et al. 2006, MNRAS, 372, 224 Beuermann, K., \& Schwope, A. D. 1994, in ASP Conf. Ser., 56, Interacting Binary Stars, ed. A. W. Shafter, 119

Bonnet-Bidaud, J. M., Mouchet, M., de Martino, D., Silvotti, R., \& Motch, C. 2006, A\&A, 445, 1037

Buckley, D. A. H., Sekiguchi, K., Motch, C., et al. 1995, MNRAS, 275, 1028

Buckley, D. A. H., Haberl, F., Motch, C., et al. 1997, MNRAS, 287, 117

Burwitz, V., Reinsch, K., Beuermann, K., \& Thomas, H.-C. 1996, A\&A, 310, L25

Chanmugam, G., \& Ray, A. 1984, ApJ, 285, 252

Cropper, M. 1986, MNRAS, 222, 225

Cropper, M. 1990, Space Sci. Rev., 54, 195

Cumming, A. 2004, in IAU Coll., 190, Magnetic Cataclysmic Variables, ed. S. Vrielmann, \& M. Cropper, ASP Conf. Ser., 315, 58

de Martino, D., Bonnet-Bidaud, J.-M., Mouchet, M., et al. 2006a, A\&A, 449, 1151

de Martino, D., Matt, G., Mukai, K., et al. 2006b, in ESA SP-604: The X-ray Universe 2005, ed. A. Wilson, 261

Duck, S. R., Rosen, S. R., Ponman, T. J., et al. 1994, MNRAS, 271, 372

Evans, P. A., \& Hellier, C. 2007, ApJ, 663, 1277

Ferrario, L., Wickramasinghe, D. T., Bailey, J., \& Buckley, D. 1995, MNRAS, 273, 17

Gaensicke, B. T., Marsh, T., Edge, A., et al. 2005, MNRAS, 361, 141

Geckeler, R. D., \& Staubert, R. 1997, A\&A, 325, 1070

Haberl, F., \& Motch, C. 1995, A\&A, 297, L37

Haberl, F., Motch, C., \& Zickgraf, F.-J. 2002, A\&A, 387, 201

King, A. R., Frank, J., \& Ritter, H. 1985, MNRAS, 213, 181

Korhonen, T., Piirola, V., \& Reiz, A. 1984, The Messenger, 38, 20

Lamb, D. Q., \& Melia, F. 1987, Ap\&SS, 131, 511

Landolt, A. U. 1992, AJ, 104, 340

Motch, C., Guillout, P., Haberl, F., et al. 1998, A\&AS, 132, 341

Norton, A. J., Butters, O. W., Parker, T. L., \& Wynn, G. A. 2007, ApJ, in press

Norton, A. J., McHardy, I. M., Lehto, H. J., \& Watson, M. G. 1992, MNRAS, 258,697

Norton, A. J., Quaintrell, H., Katajainen, S., et al. 2002, A\&A, 384, 195

Norton, A. J., Wynn, G. A., \& Somerscales, R. V. 2004, ApJ, 614, 349

Patterson, J. 1994, PASP, 106, 209

Patterson, J., Skillman, D. R., Thorstensen, J., \& Hellier, C. 1995, PASP, 107, 307

Patterson, J., Thorstensen, J. R., Vanmunster, T., et al. 2004, PASP, 116, 516

Patterson, J., Halpern, J., Mirabal, N., et al. 2006, The Astronomers Telegram, 757,1

Penning, W. R., Schmidt, G. D., \& Liebert, J. 1986, ApJ, 301, 881

Piirola, V. 1973, A\&A, 27, 383

Piirola, V. 1988, Simultaneous five-colour (UBVRI) photopolarimeter (Polarized Radiation of Circumstellar Origin), 735

Piirola, V. 1995, in Magnetic Cataclysmic Variables, ed. D. A. H. Buckley, \& B. Warner, ASP Conf. Ser., 85, 31

Piirola, V., Hakala, P., \& Coyne, G. V. 1993, ApJ, 410, L107

Piirola, V., Coyne, G. V., Takalo, S. J., et al. 1994, A\&A, 283, 163

Potter, S. B., Cropper, M., Mason, K. O., Hough, J. H., \& Bailey, J. A. 1997, MNRAS, 285,82

Rodrigues-Gil, P., Gaensicke, B. T., Hagen, H.-J., et al. 2005, A\&A, 440, 701

Rosen, S. R., Mittaz, J. P. D., \& Hakala, P. J. 1993, MNRAS, 264, 171

Schmidt, G. D., Szkody, P., Smith, P. S., et al. 1996, ApJ, 473, 483

Schwarz, R., Schwope, A. D., Staude, A., et al. 2004, ASPC, 315, 230

Schwope, A., Buckley, D. A. H., O’Donoghue, D., Hasinger, G., \& Truemper, J. 1997, A\&A, 326, 195

Shakhovskoj, N. M., \& Kolesnikov, S. V. 1997, IAUCirc, 6760, 2

Silber, A., Bradt, H. V., Ishida, M., Ohashi, T., \& Remillard, R. A. 1992, ApJ, 389,704

Southworth, J., Gaensicke, B. T., Marsh, T., et al. 2006, MNRAS, 373, 687

Southworth, J., Gaensicke, B. T., Marsh, T., de martino, D., \& Aungwerojwit, A. 2007, MNRAS, 378, 635

Staude, A., Schwope, A. D., \& Schwarz, R. 2001, A\&A, 374, 588

Staude, A., Schwope, A. D., Krumpe, M., Hambaryan, V., \& Schwarz, R. 2003, A\&A, 406, 253

Stockman, H. S., Schmidt, G. D., \& Lamb, D. Q. 1988, ApJ, 332, 282

Uslenghi, M., Tommasi, L., Treves, A., Piirola, V., \& Reig, P. 2001, A\&A, 372, L1

Vaeth, H. 1997, A\&A, 317, 476

Vaeth, H., Chanmugam, G., \& Frank, J. 1996, ApJ, 457, 407

Warner, B. 1995, Cataclysmic Variable stars (Cambridge University Press), 572

West, S. C., Berriman, G., \& Schmidt, G. D. 1987, ApJ, 322, L35

Wickramasinghe, D., \& Wu, K. 1994, MNRAS, 266, L1 